\title{
Spontaneous Skin Lesions in Beige Rats (Chediak-Higashi Syndrome of Rats)
}

\author{
Kiyokazu OZAKI, Tetsu NISHIKAWA ${ }^{1)}$, Masahiko NISHIMURA ${ }^{1)}$, and Isao NARAMA \\ Research Institute of Drug Safety, Setsunan University, 45-1 Nagaotohge-cho, Hirakata, Osaka 573-01 and ${ }^{1}$ Institute of Experimental \\ Animals, Hamamatsu University School of Medicine, Hamamatsu, Shizuoka 434-31, Japan
}

(Received 29 November 1996/Accepted 15 April 1997)

ABSTRACT. Beige rats, a model animal of Chediak-Higashi syndrome (CHS), frequently developed the skin lesions consisting of crust formations and alopecia in the skin around the neck from about 4 months of age. Erosion and ulceration were also observed in advance of the skin lesions. In severe cases, the lesions spread to all of the dorsum of the trunk. Skin tissues with or without lesions were studied histopathologically in 41 beige rats comparing with normal skin from 26 age-matched DA rats. Microscopically, epidermal lesions consisted of spongiosis, pustules and erosions with crust. Inflammatory cells in pustules consisted predominantly of eosinophil, and colonization of gram-positive cocci was occasionally observed in the surface area. Mites on the epidermis were also seen in some cases. Dermal lesions were superficial perivascular inflammatory cell infiltrations of eosinophils, neutrophils and mastocytes, and edema under the epidermal lesions. Follicles in the alopecic area showed resting stage and atrophic hair germ, but inflammatory changes were slight. Morphologic characters were very similar to those of chronic eosinophilic dermatitis or spongiotic dermatitis. — KEY WORDS: beige rat, Chediak-Higashi syndrome, dermatitis, skin.

Chediak-Higashi syndrome (CHS) is a rare autosomal, recessive genetic disease, which is characterized by the diluted color of the hair and eyes, along with increased susceptibility to infection due to the abnormal function of leukocytes containing giant granules [3,8]. CHS has been reported in mink, cattle, killer whale, cat, beige mouse and beige rat $[11-14,16,18,19]$. In contrast to sporadic occurrence of human, cattle, cat, mink or killer whale, mouse and rat cases can be obtained constantly as an inbred strain. Therefore, systemic studies on the various symptoms associated with this syndrome could be performed by using animals of these strains. The changes of the skin in human cases consisted of partial albinism of the skin and hair, and recurrent pyogenic infection by all nonspecific bacteria [2]. Similar manifestations of these skin lesions to those of human cases have been experienced in mink and cattle [17]. In contrast, beige mouse and cat have albinism, but not recurrent pyogenic infection [1]. Although the cutaneous changes in the beige rat consisted of only albinism in younger age, we found the development of severe skin lesions in the majority of adult and some aged beige rats [16]. The clinical and histopathological features of the cutaneous changes differ from those of previously reported recurrent pyogenic infection. In this paper, we reported the histopathological character of the skin lesion in the aged beige rats and discussed the possible mechanisms involved in CHS.

\section{MATERIALS AND METHODS}

Animals: Beige rats (DAbg/bg) were found as a mutant male rat with light color among DA rats $(\mathrm{DA}+/+)$ that originated from the Australian National Institute of Genetics as an inbred strain. This rat was obtained at the Institute of Experimental Animals, Hamamatsu University School of
Medicine in 1985, and since then the mutant line has been maintained by brother and sister mating under conventional housing conditions. The rats in the present study were housed in an air-conditioned animal room, fed the standard laboratory diet, and supplied water ad libitum. Forty one male and female Beige rats of 2, 4, 9, 12 and 15 months (male 25, female 16) and age-matched 26 DA rats (male 13, female 13) were used.

Histopathology: Rats were given gross examinations, anesthetized with ether, and killed by exsanguination from the abdominal aorta. Skins of all regions were stretched on a flat board, fixed in $10 \%$ phosphate-buffered neutral formalin ( $\mathrm{pH} 7.4$ ), dehydrated in a graded series of ethanol, embedded in paraffin, and $4-\mu \mathrm{m}$-thick sections were stained with hematoxylin and eosin. Representative sections were also stained by periodic acid-Schiff, Giemsa, Gram, methenamine silver nitrate, luna and phosphotungstic acid hematoxylin.

Bacterial and fungal cultures: Bacterial and fungal cultures were performed on the samples obtained from the skins of the animals. Samples from the lesions of affected beige rats and unaffected $\mathrm{DA}+/+$ rats were cultured by chocolate agar, MacConkey's agar and serum medium for common bacteria, and Sabouraud's agar medium for fungal organisms.

\section{RESULTS}

Clinical and gross findings: The coat color was paler in beige rats than in DA rats. Moreover, 15/27 beige rats of over 4 months of age developed crusts and alopecia in their skin, although these changes were not observed in all of beige rats under 2 months old and DA rats of varying ages. The earliest stage of clinically detectable skin lesions began as single or multiple small crusts up to $2 \mathrm{~mm}$ in diameter 
and as alopecia around the neck. These lesions progressed to small erosions and ulcerations. In most of cases, the lesions extended caudally to involve the skin of the postscapular dorsum. In severe cases, the lesions spread to all of the dorsum of the trunk (Fig. 1).

Bacterial and fungal cultures: Bacterial examination revealed that Staphylococcus aureus was the most commonly identified microorganism from the skin lesion of the beige rats but also from the apparently healthy skin of the DA rats and no significant differences existed between beige and DA rats. All rats were negative for fungal cultures.

Histological findings: The melanocytes of hair germs and the epidermis of beige rats contained a lower amount of melanin granules and a few abnormally giant melanosomes comparing with numerous fine granules in those of DA rats. Hair follicles and shafts were normal except for giant melanin granules in the medullary region of the hair shafts (Fig. 2).

Inflammatory changes of the skin were observed in beige rats over 4 months old. Most of the mild lesions consisted of superficial serocellular crust, spongiosis of epidermis and accumulation of eosinophils containing a few giant granules in the epidermis (eosinophilic pustule) (Fig. 3). Inflammatory changes were restricted to the surface area and follicular infundibulum. The mild to moderate lesions progressed to larger eosinophilic pustules in the epidermis, erosion or ulceration with colonization of gram-positive cocci, and were associated with superficial to middermal perivascular infiltration of inflammatory cells and edema (Figs. 4 and 5). Perivascular inflammatory cells consisted of mastocytes with a few giant granules, eosinophils, neutrophils and lymphocytes. In more severe regions, in addition to the above-mentioned lesions, the inflammatory cells infiltrated heavily and spread diffusely in the interstitium. Microabscesses containing eosinophils were rarely formed, and neutrophils emigrated into or around the wall of blood vessels were seen, but the vessel walls were not deposited with fibrinoid. Hair follicles were slightly involved in the inflammatory process except the infundibula which showed an accumulation of inflammatory cells like those in epidermis. In the alopecic area, hair follicles showed resting stage and atrophic hair germs, but folliculitis and furunculosis were rarely observed. In the chronic stage, the epidermis showed proliferative changes with moderate acanthosis, but pustules were not seen. Inflammatory cell infiltration distributed in the superficial perivascular area and vascular wall of superficial dermis was thickened by perivascular fibrosis like the "so-called onion skin lesion" (Fig. 6).

In all stages, mites on the epidermis were often seen on histological sections (Fig. 7).

\section{DISCUSSION}

In this paper, we described the morphological character of the spontaneously-occurring dermatitis that frequently
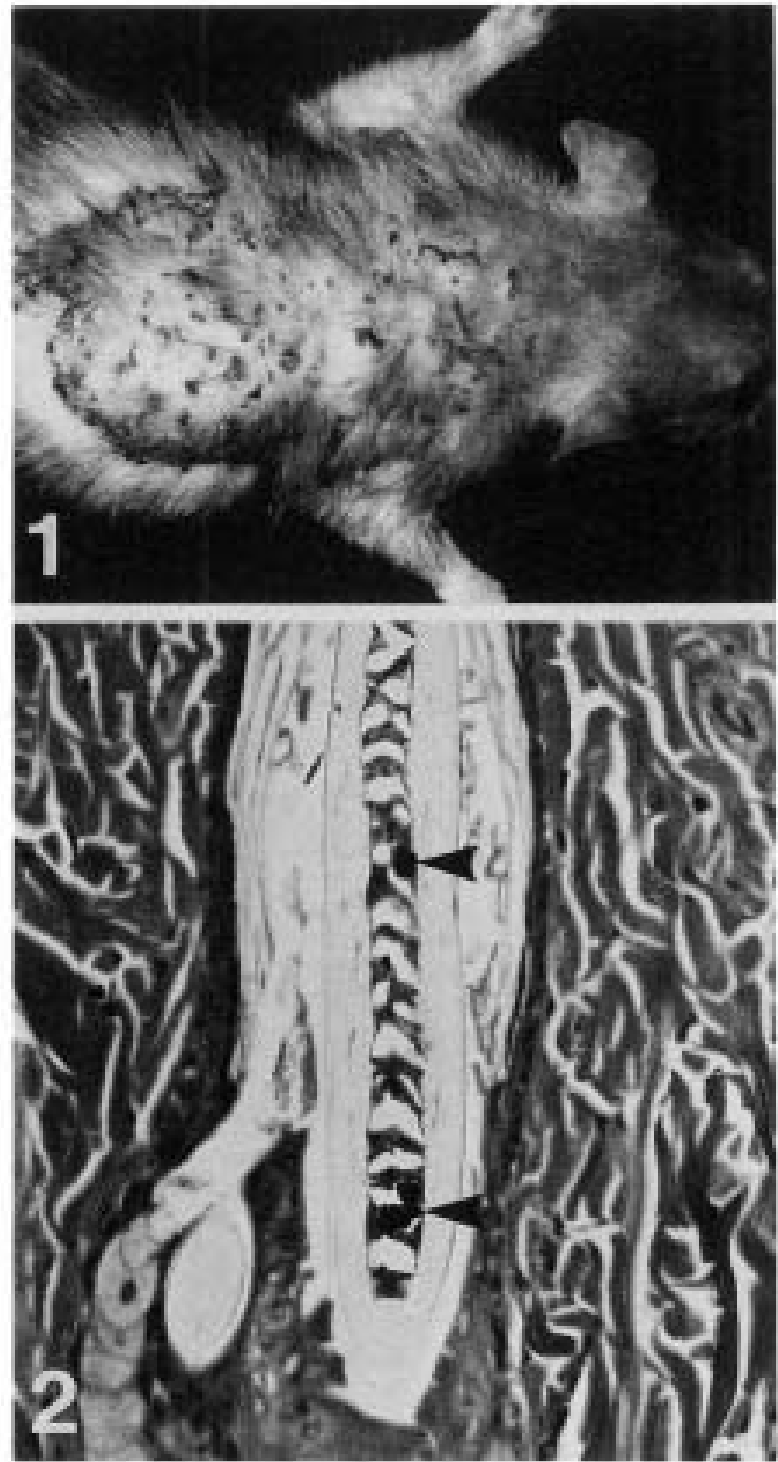

Fig. 1. Multiple small crusts and alopecia in the skin of the dorsum of trunk

Fig. 2. Normal hair shaft with a few abnormal giant melanosomes (arrowhead) in medullary region. $\mathrm{HE} \times 340$.

develops in adult and old beige rats. The most striking change in the dermatitis was the predominance of eosinophils among the infiltrated inflammatory cells in the epidermis and dermis. An increased number of mastocytes and the edematous process including spongiosis of the epidermis were also consistent features. Therefore, histological features of the spontaneous skin lesions in beige rats were very similar to those of chronic eosinophilic dermatitis or spongiotic dermatitis in morphological classification. In the pathogenesis of these dermatitides, increased vascular permeability and infiltration of eosinophils are thought to be caused by chemical mediators such as histamine contained in the mastocyte granules. 

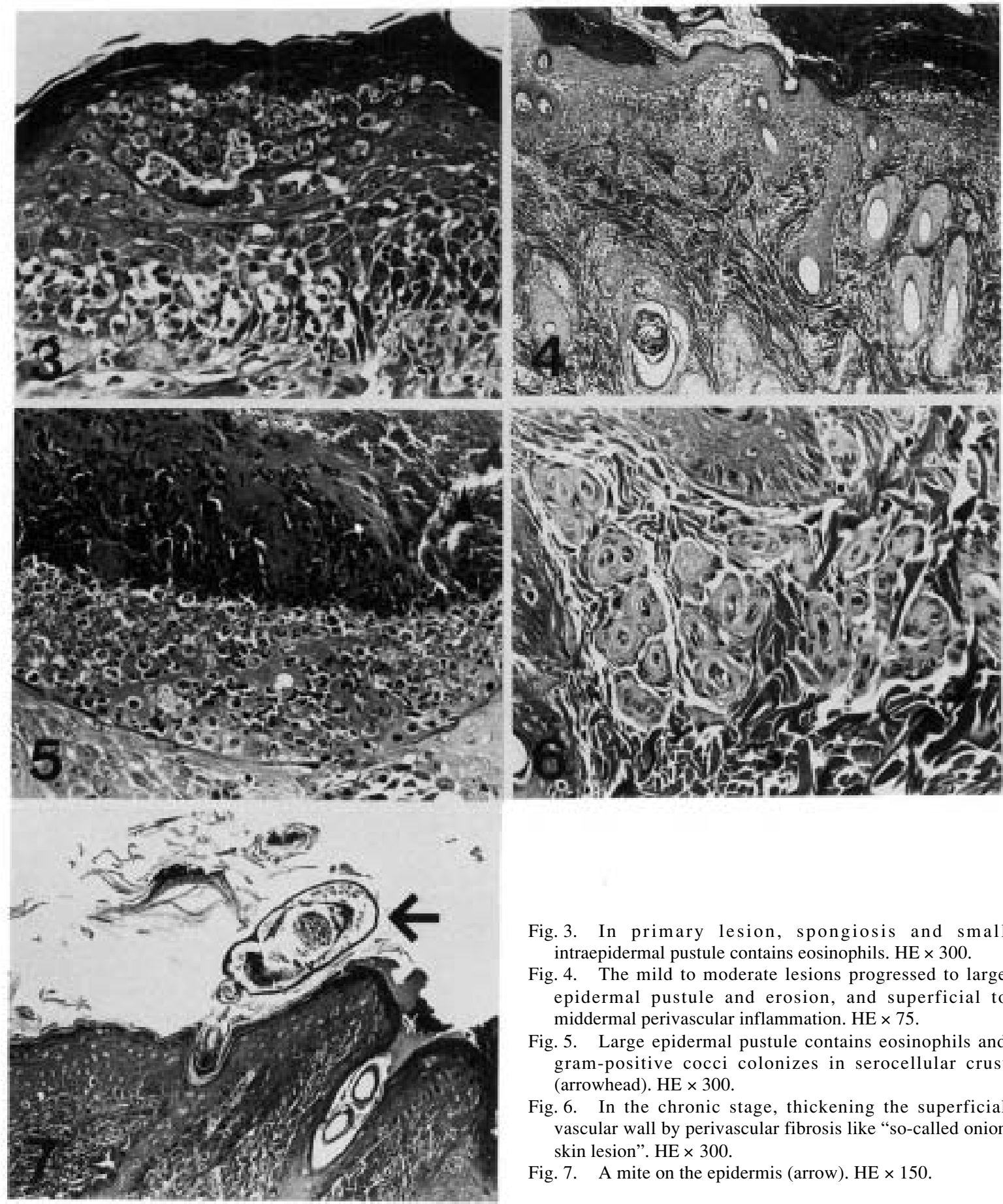

Fig. 3. In primary lesion, spongiosis and small intraepidermal pustule contains eosinophils. HE $\times 300$.

Fig. 4. The mild to moderate lesions progressed to large epidermal pustule and erosion, and superficial to middermal perivascular inflammation. $\mathrm{HE} \times 75$.

Fig. 5. Large epidermal pustule contains eosinophils and gram-positive cocci colonizes in serocellular crust (arrowhead). HE $\times 300$.

Fig. 6. In the chronic stage, thickening the superficial vascular wall by perivascular fibrosis like "so-called onion skin lesion". HE $\times 300$.

Fig. 7. A mite on the epidermis (arrow). $\mathrm{HE} \times 150$ 
Mastocytes of beige rats have been shown to release excess amounts of histamine by IgE and antigen, and to have a few giant granules [10]. Histamine release from mastocytes is closely related to type I hypersensitivity or other mechanisms to induce the degranulation of mastocytes. Supernormal histamine release from giant granules is considered to be one of the most important main factors involving skin lesions in rats. Perivascular fibrosis like "onion skin lesion" also suggested longstanding edematous process of perivascular tissue. This edematous process might also be induced by increased permeability caused by released chemical mediators from the giant granules of mastocytes. Absence of medial necrosis of the vascular wall may suggest that this edematous process was induced only by increased permeability instead of involvement in type III hypersensitivity. However, beige mice, which had similar abnormal giant granules of mastocytes and released supernormal histamine of mastocytes by IgE and antigen [1, 4], did not develop any skin lesions. Therefore, further investigation is necessary to clarify the mechanism, onset and progression of the inflammatory process.

The infestation of mites was noteworthy for the cause of dermatitis, because the distribution of the gross lesions and histopathological changes resembled those of acaric dermatitis [5]. DA rats and other strains of rats have not shown similar dermatitis under the same microbiological environmental conditions (unpublished data). Beige rats showed an increased susceptibility to parasitic infection [15]. The higher susceptibility in beige rats might be attributable to the decreased cytotoxic efficiency of eosinophils [15] that contain abnormally giant granules. Staphylococcus aureus is thought to be an indigenous bacterium and would be expected to infect existing erosional and ulcerative lesions [9]. Emigration of neutrophils in the epidermis or dermis adjacent to the ulcerative lesions in our cases might be caused by these bacteria, because neutrophilic infiltration was absent in the early lesions.

In human CHS, recurrent pyogenic infections of the skin due to dysfunction of neutrophils with giant granules is a prominent manifestation $[2,20]$. Similar manifestations with neutrophils containing giant granules were observed in cattle and mink with CHS [17, 20]. However, beige mouse and beige rat did not show recurrent pyogenic infections $[1,16]$. In beige mice and beige rats, neutrophils exhibited reduced chemotaxis in the same manner as in human, but the size of neutrophil's granules remained normal $[2,6,14]$. Thus, it was considered that the severity of pyogenic cutaneous manifestations had relation to the size of neutrophil's granule.

Alopecia with abnormal giant melanin granules of melanocyte and hair shaft were reported in color dilution alopecia of dogs [7]. The main histologic changes attributed to alopecia in these dogs were dystrophic hair follicles with twisted, irregular contours and inflammation was a secondary change [7]. Beige rats had abnormal giant melanin granules, but the structure of the hair follicle remained normal, and alopecia was caused by delayed hair regrowth suggested by the resting stage of the hair follicles and atrophic hair germs. Thus, alopecia of beige rats was not caused by color dilution alopecia but rather by secondary atrophy due to the inflammatory process.

\section{REFERENCES}

1. Bennett, J. M., Blume, R. S., and Wolff, S. M. 1969. Characterization and significance of abnormal leukocyte granules in the beige mouse: A possible homologue for Chediak-Higashi Aleutian trait. J. Lab.Clin. Med. 73: 235-243.

2. Blume, R. S. and Wolff, S. M. 1972. The Chediak-Higashi syndrome: Studies in four patients and a review of the literature. Medicine 51: 247-280.

3. Chediak, M. 1952. Nouvelle anomalie leucocytaire de caractere constitutionnel et familial. Rev. Hematol. 7: 362 367.

4. Dvorak, A. M., Hammel, I., and Galli, S. J. 1987. Beige mouse mast cells generated in vitro: Ultrastructural analysis of mutation induced by sodium butyrate and IgG-mediated, antigen-dependent degranulation. Int. Arch. Allergy Appl. Immunol. 82: 261-268.

5. Fukui, M., Matsuzaki, S., Tanaka, H., Nomura, T., Tozawa, H., and Takagaki, Y. 1961. Studies on the acaric dermatitis of albino mice in Japan. Exp. Anim. (Tokyo) 10: 83-94.

6. Gallin, J. I., Bujak, J. S., Patten, E., and Wolff, S. M. 1974. Granulocyte function in Chediak-Higashi syndrome of mice. Blood 43: 201-220.

7. Gross, T. L. and Ihrke, P. J. 1992. Dysplastic diseases of the hair follicle. pp. 298-309. In: Veterinary Dermatopathology (Gross, T. L., Ihrke, P. J., and Walder, E. J. eds.), Mosby Year Book, St. Louis.

8. Higashi, O. 1954. Congenital gigantism of peroxidase granules. The first case ever reported of qualitative abnormality of peroxidase. Tohoku J. Exp. Med. 59: 315-332.

9. Jacoby, R. O. and Fox, J. G. 1984. Biology and diseases of mice. p. 49. In: Laboratory Animal Medicine (Fox, J. G., Cohen, B. J., and Loew, F. M. eds.), Academic Press, Orlando.

10. Jippo-Kanemoto, T., Kasugai, T., Yamatodani, A., Ushio, H., Mochizuki, T., Tohya, K., Kimura, M., Nishimura, M., and Kitamura, Y. 1993. Supernormal histamine release and normal cytotoxic activity of (Chediak-Higashi) rat mast cells with giant granules. Int. Arch. Allergy Immunol. 100: 99106.

11. Kramer, J. W., Davis, W. C., and Prieur, D. J. 1977. The Chediak-Higashi syndrome of cats. Lab. Invest. 36: 554-562.

12. Lutzner, M. A., Lowrie, C. T., and Jordan, H. W. 1967. Giant granules in leukocytes of the beige mouse. J. Hered. 58: 299300 .

13. Lutzner, M. A., Tierney, J. H., and Benditt, E. P. 1966. Giant granules and widespread cytoplasmic inclusion in a genetic syndrome of Aleutian mink. Lab. Invest. 14: 2063-2079.

14. Nishimura, M., Inoue, M., Nakano, T., Nishikawa, T., Miyamoto, M., Kobayashi, T., and Kitamura, Y. 1989. Beige rat: A new animal model of Chediak-Higashi syndrome. Blood 74: 270-273.

15. Nishimura, M., Nishikawa, T., Ashizawa, O., Ishii, A., Yoshida, T., and Kitamura, Y. 1990. Susceptibility to infection in beige (Chediak-Higashi sundrome) rat. Nihon Shikkan Moderu Dobutsu Kenkyukai Kiroku 6: 74.

16. Ozaki, K., Maeda, H., Nishikawa, T., Nishimura, M., and 
Narama, I. 1994. The Chediak-Higashi syndrome of rats: Light and electron microscopic characterization of abnormal granules in beige rats. J. Comp. Pathol. 110: 369-379.

17. Padgett, G. A., Reiquam, C. W., Gorham, J. R., Henson, J. B., and O'Mary, C. C. 1967. Comparative studies of the Chediak-Higashi syndrome. Am. J. Pathol. 51: 553-571.

18. Taylor, R. F. and Farrell, R. K. 1973. Light and electron microscopy of peripheral blood neutrophils in a killer whale affected with Chediak-Higashi syndrome. Fed. Proc. 32: 822a.

19. Umemura, T., Katsuta, O., Goryo, M., Hayashi, T., and Itakura, C. 1983. Pathological findings in young Japanese black cattle affected with Chediak-Higashi syndrome. Jpn. J. Vet. Sci. 45: 241-246.

20. Weary, P. E. and Bender, A. S. 1967. Chediak-Higashi syndrome with severe cutaneous involvement. Arch. Intern. Med. 119: 381-386. 